\title{
Implementing and Operating Computer Graphics in the Contemporary Chemistry Education
}

\author{
Olga Popovska \\ Faculty of Technological Sciences \\ "Mother Teresa" University, 12 Udarna Brigada 2a, 1000 Skopje, Republic of Macedonia
}

\begin{abstract}
Technology plays a crucial role in modern teaching, providing both, educators and students fundamental theoretical insights as well as supporting the interpretation of experimental data. In the long term it gives students a clear stake in their learning processes. Advancing in education furthermore largely depends on providing valuable experiences and tools throughout digital and computer literacy. Here and after, the computer's benefit makes no exception in the chemistry as a science. The major part of computer revolutionizing in the chemistry laboratory is with the use of images, diagrams, molecular models, graphs and specialized chemistry programs. In the sense of this, the teacher provides more interactive classes and numerous dynamic teaching methods along with advanced technology. All things considered, the aim of this article is to implement interactive teaching methods of chemistry subjects using chemistry computer graphics. A group of students $(n=$ 30 ) at the age of 18-20 were testing using methods such as brainstorming, demonstration, working in pairs, and writing laboratory notebooks. The results showed that demonstration is the most acceptable interactive method $(95 \%)$. This article is expected to be of high value to teachers and researchers of chemistry, implementing interactive methods, and operating computer graphics.
\end{abstract}

Keywords: brainstorming; chemistry education; demonstration; molecular visualization.

\section{Introduction}

The traditional teaching methodologies are becoming obsolete in the tech- world that encourages students to think critically and creatively. It is very likely that the new generations of undergraduate students increasingly differ from the traditional students the teachers used to teach. It affects how and by which means students learn over the coming decades. Therefore, the new generations of young people expect to learn quickly and tend to have less patience for studying textbooks. Although some textbooks offer three-dimensional photographs of molecular models with shadowing and colours, they cannot match what modern visualization technologies have to offer ${ }^{1}$.

The traditional ways of developing new methods and procedures in chemistry require a lot of trial and error and may also impact the environment. To expedite the research process of determining the correlation between the structure and properties and prediction of the properties of new materials, researchers today often stand for computer simulations, which in turn are made possible by the modern computer graphics and visualization hardware. Additionally, computer simulations, being

*Corresponding author: Olga Popovska

Email address: olga popovska@yahoo.com

DOI: http://dx.doi.org/10.13171/mjc65/01711241030-popovska executed in virtual computer environments are a great way to lessen the burden of chemical developments on the environment.

On the other hand, chemistry research today focuses more and more on phenomena that are well understood and communicated by the means of visual representations. For example, research on carbon nanotubes, conducting polymers, and selfassembling materials is carried out and communicated by the help of computer-generated images ${ }^{2,3}$. One of the main tasks in teaching chemistry is how to present the microscopic world acceptable to the students. In the digital era, computer graphics enables the visualization of chemistry phenomena to be a part in the chemistry education. The fact that tasks can be written and can be digitally stored on high-resolution graphics displays, makes the look of the laboratory to be more sophisticated than ever before. The digital visualization in the chemistry laboratory is focused towards the student: students can now develop spatial thinking skills of macroscopic and microscopic world, and relate them to theory.

Thus, the contemporary development of computer hardware and software and their visualization capabilities fundamentally influence all

Received October 31, 2017

Accepted, November 8, 2017

Published November 24, 2017 
major areas of chemistry: education, research and environmental impacts. Visualization tools and highperformance computing have changed the nature of chemistry research and provide the commitment to transform chemistry instruction as well.

Visualizing objects in alternative ways improves the students' abilities and strengthens their spatialthinking skills. Student beginners in chemistry can now be taught using a wide range of molecular visualizations: structural formulas, line drawings, physical models, and a variety of dynamic threedimensional computer-generated molecular models. Computational chemistry is used with a mathematical approach which is sufficiently well developed so that it can be automated on a computer $^{2}$.

The chemical problems could be solved with the use of chemical, mathematical, or numerical calculations. Molecular-modelling programs are essential in the study and analysis of 3D biomolecular structures such as proteins and nucleic acids, due to the notably complex structure of the functional biomolecules. The computer graphics applied to crystallography were used by ${ }^{3}$ to show an animation of cubic crystalline structures to visualize the lattice and keep the student attention focused on the teaching material.

The computer laboratory should be equipped with different kinds of technology, including multimedia, modern computer graphics workstations and diverse chemistry software packages. In this regard, the level of competence of the students should be taken into account when equipping laboratories in order to assure gradual exposition to involved procedures and complex equipment. The modern graphics are important in order to create useful chemistry information based on either dynamic molecular animations or making a dynamic presentation of how a piece of equipment is to be used $^{4}$.

With the use of movies, the students are meant to excel the understanding of the issue versus the teachers who are required to adapt to such changes in the classroom. A suitable choice of instructional images or movies changes the perspective on education and promise to improve students' visualization skills. The preparation for the experiment or demonstration of the lesson can be easily understood with the use of the visualization in chemistry movies ${ }^{3}$. This is important for students to<smiles>c1ccc2ccccc2c1</smiles>

a

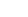

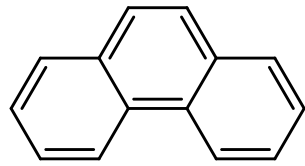

b understand difficult concepts of the electron configuration and structural modifications and general chemistry. In terms of molecular visualization tools in the education system, students should be able to interpret the images they produce. The application of modern computer graphics enables better visualization of the structures, and also helps better understanding the stereochemistry and organic chemistry, binding bonds and tridimensional configuration ${ }^{5}$. It also helps chemists to be deeper prepared for making observations before running into the actual experiments.

In this article, the focus is on computational chemistry, which in turn is the basis of organic chemistry, stereochemistry, and biochemistry. In particular, the molecular modelling tools are useful in helping students understanding concepts in chemistry settings. This article offers also recommendations and perspectives on the training of chemistry teachers in cheminformatics using interactive teaching methods.

\section{Computational Chemistry Software}

\section{Model Structures}

The prime and important thing in chemistry, especially in organic chemistry and biochemistry, is the ability to represent $3 \mathrm{D}$ molecules in space ${ }^{6}$. Unfortunately, many students are incapable to visualize in space and have difficulties in resolving the structure of the substance ${ }^{1}$. The possibilities to represent the structures either in two- or threedimensional representation are important to comprehend the bonds, isomerism, electronic configurations, and also the atom arrangements in particular molecules ${ }^{7}$.

The modelling of chemical structures is essential to almost every chemical software package ${ }^{4}$. Fundamentally to the modelling is the implementation of the theoretical knowledge in the software in order to help students better understanding the principles of the microscopic world and the ways atoms works within it ${ }^{8,9}$.

Fig. 1 shows models of polycyclic aromatic hydrocarbons (I): naphthalene (a), phenanthrene (b), and anthracene (c) in rendering with wireframe (II) and space fill (III). This kind of representation is introduced to help understand $3 \mathrm{D}$ arrangements of the atoms.

$$
\text { b }
$$<smiles>c1ccc2cc3ccccc3cc2c1</smiles>

C 


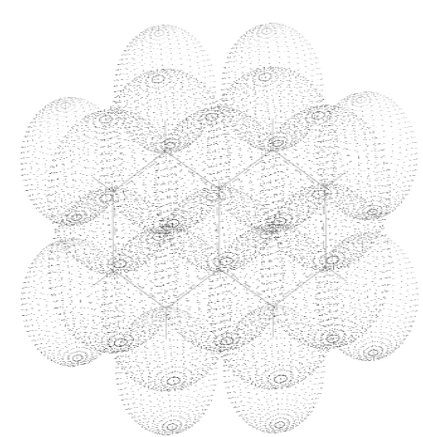

a

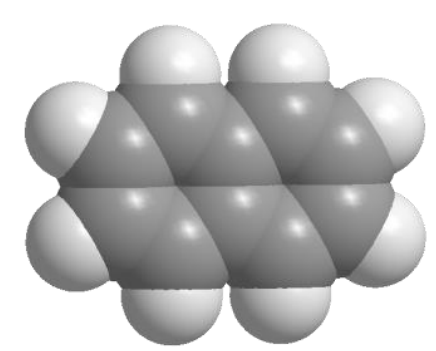

a

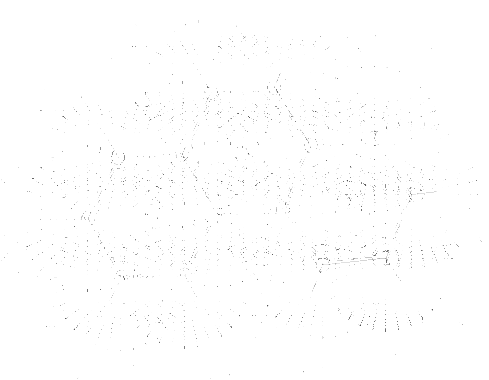

b

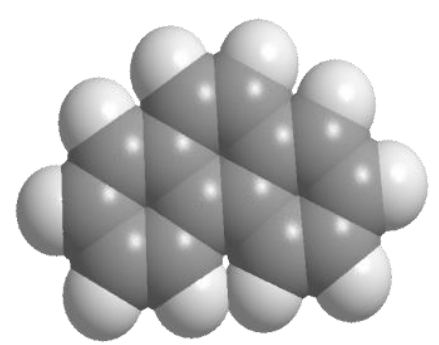

$\mathrm{b}$

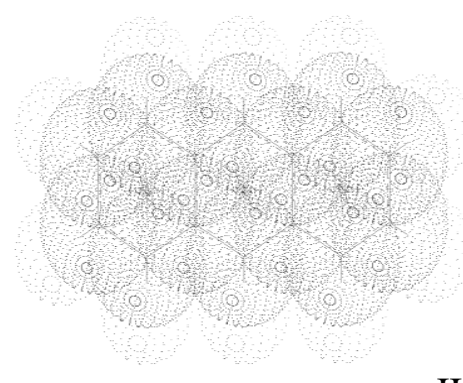

C

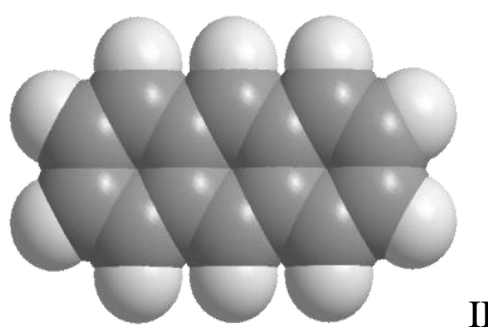

C
III

Figure 1. Structural formulas of naphthalene (a), phenanthrene (b), and anthracene (c)

\section{Programs for Drawing Laboratory Equipment}

One of the most important tasks for the young researchers in tackling the challenges of chemistry is in the preparation of laboratory notebooks ${ }^{1}$. The main lab activity lays in understanding the use of various equipment pieces and making accurate interconnections among them so that complete experiments can be accomplished successfully. In order to obtain a successful outcome during the experimental part, numbers of steps should be executed properly.
In their laboratory notebooks, students are theoretically supposed to take good care in drawing pieces of equipment in order to reproduce the experimental setup accurately in future. They should draw diligently and always bear in mind that the laboratory notebook is an important part in upgrading their knowledge ${ }^{2,10,11}$. There are various chemical programs such as ACD/ChemSketch* and ChemDraw in which ready-made templates of different pieces of laboratory equipment are applied.

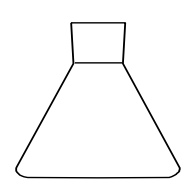

a

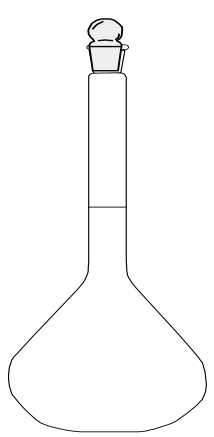

e

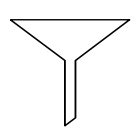

b

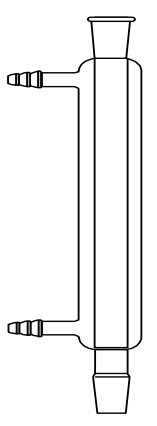

f
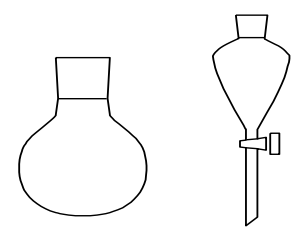

c
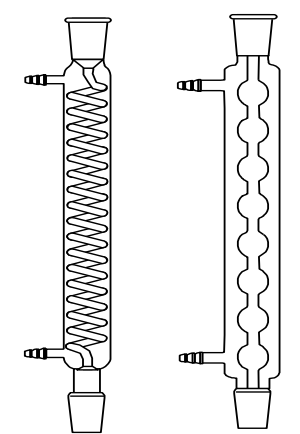

g d

$\mathrm{h}$

Figure 2. Laboratory equipment 
Chemsketch $^{*}$ is a molecular modelling program used to create and modify images of chemical structures. Displaying molecules in two- and threedimensional rendering helps in understanding the structure of chemical bonds and the nature of the functional groups. The program offers advanced features such as rotation of molecules and application of colours in order to improve visualization. The students can easily choose the proper flask, apparatus for distillation or extraction and to put in the suitable experimental setup. This is a great opportunity for students to retain a record of the laboratory setup and to achieve better results in the experimental work of chemistry ${ }^{3}$.

Fig. 2 shows drawings of the most commonly used pieces of laboratory equipment: Erlenmeyer flask (a), funnel (b), round-bottom flask (c), separatory funnel (d), volumetric flask (e), condensers: Liebig (f), Grahams (g), and Allihn (h).

\section{Chemistry Education}

Educational researchers tend to implement new possibilities for teaching in order to make connection between the virtual and the real world. Trends in chemistry teaching tend to include simultaneously, the position of the students on one hand and how well they understand the chemistry and on the other hand, the role of the teacher in the aim to improve teaching methods and to introduce new methodological approaches ${ }^{12}$.

Recognizing the quality of learning in almost all exact science courses, including chemistry, is that all steps in learning are equally important, from equations, formulas, and structure representations to making connections between the practical experience gained in the laboratory and the theoretical knowledge. Efficient comprehension of concepts in chemistry depends on how good the experiments are made, continuously improving the processes, application of results to related fields, and designing experiments around less toxic or irritable substances ${ }^{1}$. Modern chemistry is a highly complex and interdisciplinary subject, related to molecular biochemistry, medicine, pharmacy and other fields, and as such, many times face unpredictable situations in solving problems at the molecular level. The reactions and the arrangement of the atoms in many complex structures are difficult to grasp, not only for the students, but also for beginner researcher. The design of new molecules, resolving the structure of substances, calculations, modeling of compounds etc., are all important tasks in modern chemistry. In addition, many forensic cases, processing of spectroscopic data and all modern laboratory equipment are connected with the use of computers where the analysis should be completed in a reasonable time and manner frame ${ }^{10-13}$.

\section{Computers in Chemistry Education}

Understanding the way computer systems works in the chemistry education is as important as understanding chemistry itself. Laboratory computer systems support various activities in a chemistry laboratory. Some systems are used to perform preprogrammed mathematical calculations, or are embedded systems in the laboratory instrumentation with specialized firmware for drawing or comparing spectra, determining concentration, calculating the kinetics of the reaction etc. Others are interactive graphical systems that are used in the students' exercises. Such setups help the chemistry laboratory appears as a place where students can practice their theoretical knowledge and upgrade their motor and spatial skills ${ }^{14}$.

On the other hand, there are also programs which help students to better understand the principles at the molecular level and to organize the results in the laboratory logs. Drafting the problem statement in chemistry education is equally important as understanding how to solve it. The chemical software packages are useful in shortening the time for the calculations, visualizing the molecular structures, resolving certain problems in the structures etc.

\section{Software Packages}

There are different kinds of software packages, either free or commercially available. Most of these software packages are easy to install by the end users and cover a wide range of applications in chemistry research and education ${ }^{13}$. Most programs are able to deal with various file formats and also offer export/import possibilities including high-quality vector graphics formats such as wmf, emf, and pdf 12,13 .

The chemistry as science has different disciplines such as theoretical and applied organic chemistry, stereochemistry, crystal chemistry etc. Among the main activities in most chemical disciplines are drawing complex structures, resolving and assignment of spectral data, and designing structures in order to save reagents in real experiments. Today's personal computers are already able to cope with most of the technical difficulties posed in front of a chemistry student or researcher. Teaching and learning methods are supported by today's computational capabilities and the specialized software packages to be installed on the computer. Combining different chemistry programs allows to better predict the behavior of certain substances, and to have a better presentation on how the molecules function at a microscopic level.

The task of choosing software for a chemistry laboratory is complex in the way that a number of 
criteria have to be considered in order to make the right decisions. It mainly depends on the age of the students and whether the software is aimed for students in the primary, secondary or tertiary level. Due to this, it is important which software interface correspond the students'foreknowledge. Further, different software packages have different levels of accuracy so that the criteria of choosing the level of accuracy should be in accordance to the requirements of the task handed. And finally, the organization of the curriculum has to be taken into account in order to arrive at a useful and wellrounded laboratory setup.

\section{Stereochemistry}

Stereochemistry as a sub-discipline of chemistry deals with the structure of molecules in three<smiles>OC[C@H]1C[C@H](O)[C@H](O)[C@H](O)[C@H]1O</smiles>

$\alpha$-D-glucopyranose dimensions. Stereoisomers, molecules with the same formula, but differ in three-dimensional orientations of their atoms in space are the main studies of stereochemistry ${ }^{14}$. Stereoisomers are divided into two groups: with molecules that are mirror images of each other, and those with molecules that are not mirror images of each other. Enantiomers are stereoisomers that are mirror images, while diastereoisomers are those enantiomers that are not mirror images. On the other hand, E-Z isomers and cis-trans isomers are also diastereoisomers. Enantiomers are said to be chiral with the presence of an asymmetric carbon center. Biomolecules and drugs are optically active isomers where one structure is active ${ }^{15}$.

Fig. 3 presents the structures of two types of sugars in their three-dimensional configuration.<smiles>OC[C@H]1C[C@H](O)[C@H](O)[C@H](O)[C@H]1O</smiles>

$\alpha$-D-gulopyranose

Figure 3. Stereo projections of $\alpha$-D-glucopyranose and $\alpha$-D-gulopyranose with ACD/ChemSketch

The handedness whether right or left is present in the individual enantiomers. Most amino acids are levorotatory (L) while sugars are dextrorotatory (D). An equimolar mixture of two enantiomers is called a racemic mixture. The possession of zero net rotation of plane-polarized light is a result of the mixture that contains equal numbers of the dextrorotatory and levorotatory molecules. According to the presence of two chiral centers, there are four isomers of ketoconazole (Fig. 4), marked with an asterisk (*). The structure (I) is responsible for the desired physiologic effects, while the other enantiomer (II) is less active, inactive, or sometimes even productive of adverse effects.

I

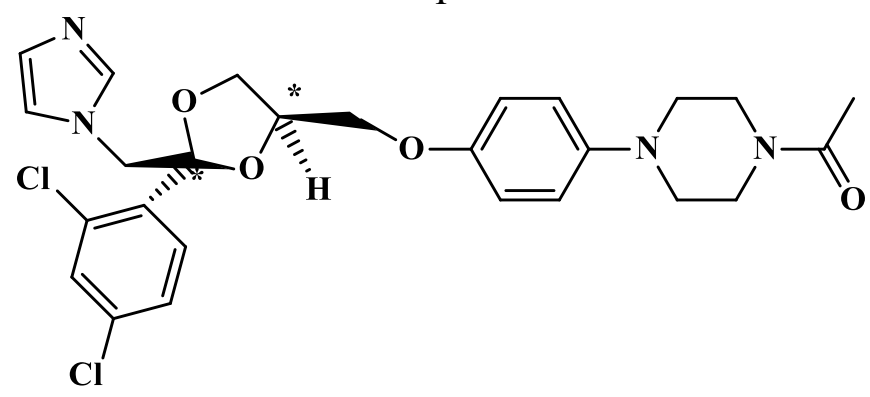

II

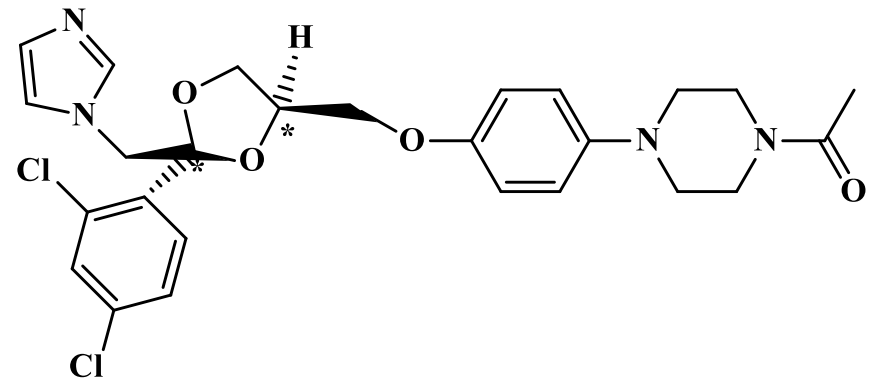




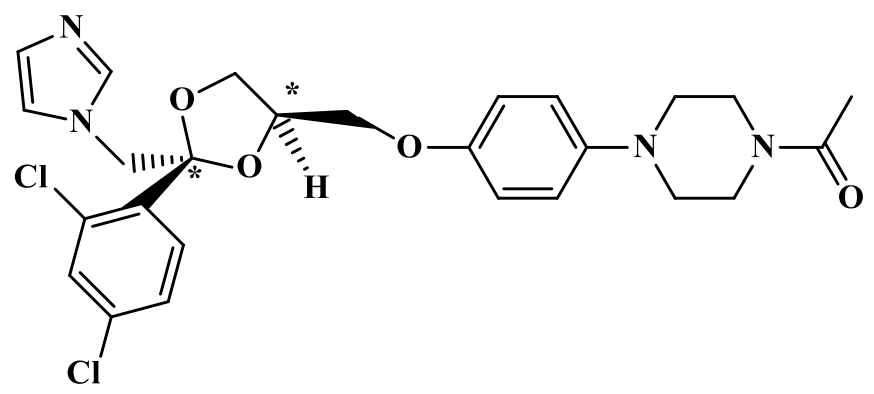

IV

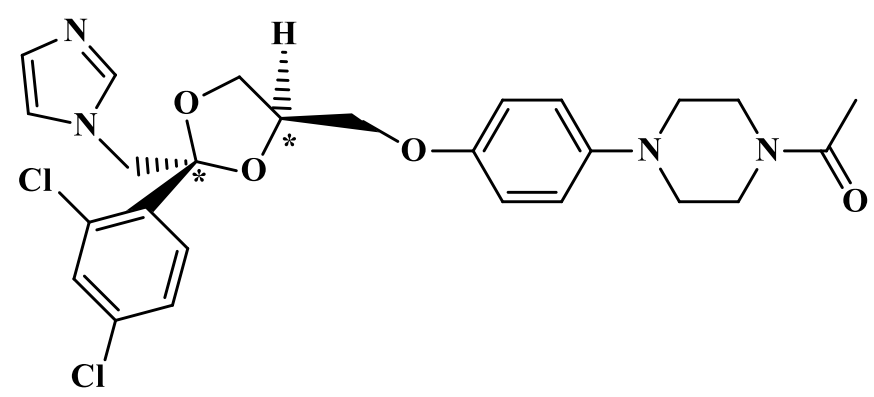

Figure 4. Stereoisomer structures of ketoconazole

A large number of drugs are chiral but are sold as racemates. The significance of studying chirality in the biological world is due to the fact that one of the two enantiomers can be metabolized by the particular organism. Thus, enantiomerically pure drugs are found to be more effective than the racemic mixture. The application of computer graphics surrounds in molecular modeling methods including theoretical and computational. A less time consuming and eco-friendly method can be developed in order to investigate the structure of the substances, the stability, and also molecular recognition of specific molecules.

Teaching materials in stereochemistry deal with the conditions under which certain phenomena at the molecular level directly affect the macroscopic behavior of the substances. The computational graphics is useful in studying chemistry and it is important for teachers to be up-to-date with the software package and hardware.

\section{Contemporary methods for interactive teaching}

The contemporary teaching includes interactive methods such as brainstorming, working in pairs, individual exercises, demonstration, association, and writing laboratory notebook ${ }^{16}$. This means that teachers should upgrade their teaching skills in order to improve the educational process.

One of the most common method, brainstorming provides deductive conclusion of certain topic and it also gives opportunity to combine and to develop new ideas. The methods which include exercises for pairs or individual working are designed to step forward to the frontal teaching way in the organization in the classroom, typically for the traditional teaching method. These methods are involved in order to provoke individual thinking and the teachers can easily follow students' work ${ }^{16}$.

The method of demonstration is one of the most usefulness methods in experimental work in the lack of either apparatus or substances. The teacher can demonstrate the experiment in front of the students to point how to be more successfully in their attempts and what kind of precautions should be taken designing the experiment. The demonstration can be easily performed on a computer by the teacher showing to the students how some software program works. The association method is efficacy in increasing the visualization capacity of students using simulating movements of molecules in chemistry software program. The students can see the structural formulae in 3D which are displayed on the computer ${ }^{1}$. The idea is to present their own conclusions about functional groups, chemical bonds, and marked atoms using 3D structures with the program. The laboratory book is worth to mention as a good practice to develop student's thinking and to repeat the exercise according to the written notice.

\section{Study Participants}

The research was conducted where 30 students, aged 18-20 years took part in the analysis. The analysis was in a chemistry laboratory equipped with basic laboratory equipment and computers with chemistry software package. The collection data were depended on implementing interactive teaching methods. The methods changed randomly in the accordance of the topic which was studied. 


\section{Data Analysis}

The data from the research showed that students are always ready to accept new methodological approaches, but they must be well defined and organized. The relationship between students was valuable information in achieving better results. Some difficulties were present with the respect to complex structure of the substance.

For example, the brainstorming interactive method involved some difficulties when its successfully application was observed. The students who had better foreknowledge knew the answers and the ideas, while the students who were with less knowledge on certain topic in chemistry told many words, but sometimes they were very distance from chemistry.

On the other hand, students memorized better when the main terminology in certain branch of chemistry is linked with association (71\%) either it is written with certain colour in the specialized chemistry program or it is linked indirectly with the tools in the program. 3D presentations in chemistry program help students to understand easily complex chemistry material. The young researchers can express their ideas easily when they work in groups $(64 \%)$ and less if they work individually $(56 \%)$. The cooperation in pairs is of great importance in order to develop supporting links in solving chemistry tasks. The first thing the students should learn when they work in pairs is to know how to communicate. The matching partner is the main key whether the collaboration would be successfully or not. If both students are equally intelligent and are good students, it is more difficult such a pair to continue to cooperative in the future. The result is more satisfactory if such a pair works individually.

The interactive method which involves demonstration was acceptable for almost every student $(95 \%)$. This showed that students felt more comfortable when the instructor (teacher) showed them unequivocally how to improve their motor and spatial skills, performing the experiment in front of the students.

The students found writing in laboratory notebook boring activity because everything nowadays is digitalized. This is a worth idea in further research to focus how to store their own results safe, not using paper, but still be available every time they look for the information and last for longer time.

\section{Conclusion}

In this article, the applications of computer graphics have been considered in contemporary chemistry education over several branches such as organic chemistry, biochemistry, and stereochemistry. Several visualization tools were useful and applicable to drawing molecular models and laboratory equipment that can be displayed on high resolution screens. The unquestionable usefulness of computer systems in the present day is that educational settings are a strong incentive to computer programmers to continue their efforts for further development and improving of the software. The connections between chemistry and other branches of science is clearly in hope that it will be useful to students, teachers and researches in making decisions on software usage and solving various problems in chemistry. In addition, the contemporary teaching methods can be successfully implemented in the teaching chemistry where the focus is towards students.

\section{Acknowledgement}

I owe A. C. sincere gratitude for the contribution to this article giving constructive ideas and suggestions.

\section{References}

1- H. J. Fan, J. Heads, D. Tran, N. Elechi, Teaching chemistry with computers. Int. J. Inf. Educ. Technol., 2015, 5, 184-188.

2- J. Lee, L. W. Kang, H. S. Kim, J. I. Kim, CoCoot: a real-time collaborative tool for biomolecular modeling and visualization. IEEE International Symposium on Virtual Reality Innovation, 2011, 281-286.

3- B. Pekdag, J. F. Le Marèchal, Movies in chemistry education. APFSLT, 2010, 11, 1-19.

4- I. V. Tetko, J. Gasteiger, R. Todeschini, A. Mauri, D. Livingstone, P. Ertl, V. A. Palyulin, E. V. Radchenko, N. S. Zefirov, A. S. Makarenko, V. Y. Tanchuk, V. V. Prokopenko, Virtual computational chemistry laboratory design and description. J. Comput. Aided Mol. Des., 2005, 19, 453-463.

5- L. L. Jones, K. D. Jordan, N. A., Stillings, Molecular visualization in chemistry education: the role of multidisciplinary collaboration. Chem. Educ. Res. Pract., 2005, 6, 136-149.

6- Y. He, B. Wang, R. K. Dukor, L. A. Nafie, Determination of absolute configuration of chiral molecules using vibrational optical activity: a review. Appl. Spectrosc., 2011, 65, 699-723.

7- D. Nori-Shargh, S. Asadzadeh, F. R., Ghanizadeh, F. Deyhimi, M. M. Amini, $\mathrm{S}$. Jameh-Bozorghi, $\mathrm{Ab}$ initio study of the structures and dynamic stereochemistry of biaryls. J. Mol. Struct: Theochem, 2005, 717, 41-51.

8- S. Bottomley, D. Chandler, E. Morgan, E. Helmerhorst, AMVLE, A new integrated molecular visualization learning environment, Biochem. Mol. Biol. Educ., 2006, 34, 343-349. 
9- J. E. Stone, J. Saam, D. J. Hardy, K. L. Vandivort, W. W. Hwu, K. Schulten, High performance computation and interactive display of molecular orbital on GPUs and multi-core CPUs, Conference: Proceedings of 2nd Workshop on General Purpose Processing on Graphics Processing Units, 2009, 9-18.

10- M. Jaskolski, M. Gilski, Z. Dauter, A. Wlodawer, Stereochemical restraints revisited: how accurate are refinement targets and how much should protein structures be allowed to deviate from them? Acta Crystallogr. D, 2007, 63, 611-620.

11- A. T. Gabriel, T. Meyer, G. Germano, Molecular graphics of convex body fluids. J. Chem. Theory Comput., 2008, 4, 468-476.

12- H. K. Wu, P. Shah, Exploring visuospatial thinking in chemistry learning. Scie. Educ., 2004, 88, 465-492.

13- M. Kaushik, M. (2014). A review of innovative chemical drawing and spectra prediction computer software. Mediterr. J Chem., 2014, 3, 759-766.

14- T. Cieplak, J. L. Wisniewski, A new effective algorithm for the stereochemical characteristics of compounds during their registration in databases, Molecules, 2001, 6, 915-926.

15- F. Long, R. A. Nicholls, P. Emsley, S. Gražulis, A. Merkys, A. Vaitkus, G. N. Murshudov, AceDRG: a stereochemical description generator for ligands. Acta Crystallogr. D Structur. Biol., 2017, 73, 112-122.

16- E. Tomevska-Ilievska, Interactive approaches in teaching. Ss. Cyril and Methodius University, 2015. 